PROCEEDINGS OF THE

AMERICAN MATHEMATICAL SOCIETY

Volume 136, Number 10, October 2008, Pages 3591-3600

S 0002-9939(08)09347-7

Article electronically published on May 8, 2008

\title{
UNIFORM HYPERBOLICITY FOR RANDOM MAPS WITH POSITIVE LYAPUNOV EXPONENTS
}

\author{
YONGLUO CAO, STEFANO LUZZATTO, AND ISABEL RIOS
}

(Communicated by Jane M. Hawkins)

\begin{abstract}
We consider some general classes of random dynamical systems and show that a priori very weak nonuniform hyperbolicity conditions actually imply uniform hyperbolicity.
\end{abstract}

\section{INTRODUCTION AND STATEMENT OF RESULTS}

In this paper we consider smooth random dynamical systems $F$ over an abstract dynamical system $(\Omega, \mathcal{F}, \mathbb{P}, \theta)$, where $(\Omega, \mathcal{F}, \mathbb{P})$ is a complete probability space and $\theta: \Omega \rightarrow \Omega$ is a $\mathbb{P}$ preserving ergodic invertible transformation. More specifically, we have a skew-product

$$
F: \Omega \times M \rightarrow \Omega \times M
$$

given by

$$
F(\omega, x)=\left(\theta(\omega), \phi_{\omega}(x)\right),
$$

where $M$ is a compact manifold endowed with a Riemannian metric which induces a norm $|\cdot|$ on the tangent space and a volume form that we call Lebesgue measure. Throughout the paper we suppose that for $\mathbb{P}$ - a.e. $\omega$,

$$
\phi_{\omega}: M \rightarrow M
$$

is a $C^{1}$ local diffeomorphism. We let

$$
\left|D \phi_{\omega}\right|=\sup _{x \in M}\left|D_{x} \phi_{\omega}\right| \text { and }\left|D \phi_{\omega}^{-1}\right|=\sup _{x \in M}\left|D_{x} \phi_{\omega}^{-1}\right|
$$

and assume standard integrability conditions

$$
\int_{\Omega}\left|D \phi_{\omega}\right| d \mathbb{P}<\infty \text { and } \int_{\Omega}\left|D \phi_{\omega}^{-1}\right| d \mathbb{P}<\infty .
$$

Notice that these conditions are not automatic since we do not assume that $\phi_{\omega}$ depends continuously on $\omega$ in any way.

Received by the editors April 2, 2007, and, in revised form, September 3, 2007.

2000 Mathematics Subject Classification. Primary 37H15.

Key words and phrases. Skew-product, random maps, Lyapunov exponents.

The first author was partially supported by NSFC(10571130), NCET, and SRFDP of China and the Royal Society.

The second author was partially supported by EPSRC grant GRT0969901.

The third author was partially supported by CAPES and FAPERJ (Brazil). The authors would like thank M. Benedicks and M. Viana for their suggestions and encouragement.

(C)2008 American Mathematical Society Reverts to public domain 28 years from publication 
Random maps of this kind have been extensively studied from various points of view, such as the existence and properties of invariant measures and equilibrium states [7, 2], and the continuity properties of the entropy [11]; see [8, 10] for an extensive survey and references. Many results depend on some hyperbolicity of the random maps or, in the language of skew-products, on some hyperbolicity in the fibres. Our main goal in this paper is to show that in several cases, uniform hyperbolicity estimates can be obtained from some a priori strictly weaker nonuniform hyperbolicity assumptions.

\subsection{Basic definitions.}

1.1.1. Random continuous functions. A function $f: \Omega \times M \rightarrow R$ is a random continuous function if

(1) $x \mapsto f(\omega, x)$ is a continuous function for a.e. $\omega \in \Omega$;

(2) $\omega \mapsto f(\omega, x)$ is measurable for all $x \in M$;

(3) $\omega \mapsto \sup _{x \in M}|f(\omega, x)|$ is integrable with respect to $\mathbb{P}$.

1.1.2. Topology on the space of measures. We let $\mathcal{M}_{\mathbb{P}}(F)$ denote all $F$-invariant probability measures on $\Omega \times M$ whose marginal on $\Omega$ coincides with $\mathbb{P}$ (such measures can be characterized in term of their disintegrations $\mu_{\omega}$ by $\phi_{\omega}\left(\mu_{\omega}\right)=\mu_{\theta \omega}$ a.s. $)$. We equip $\mathcal{M}_{\mathbb{P}}(F)$ with the smallest topology such that

$$
\nu \rightarrow \int_{\Omega} \int_{M} f(\omega, x) d \mu_{\omega}(x) d \mathbb{P}(\omega)=\int_{\Omega \times M} f(\omega, x) d \mu(\omega, x)
$$

is continuous for every random continuous function $f$. We let

$$
\mathcal{E}_{\mathbb{P}}(F) \subset \mathcal{M}_{\mathbb{P}}(F)
$$

denote the subset of ergodic measures.

1.1.3. Fibrewise Lyapunov exponents. For $\omega \in \Omega$, let $\phi_{\omega}^{(0)}$ be the identity map on $M$ and, for $k \in \mathbb{N}$, define $\phi_{\omega}^{(k)}$ by

$$
\phi_{\omega}^{(k+1)}=\phi_{\theta^{k}(\omega)} \circ \phi_{\omega}^{(k)} .
$$

Then we can define a family of iterates of $F$ by

$$
F^{n}(\omega, x)=\left(\theta^{n}(\omega), \phi_{\omega}^{(n)}(x)\right) .
$$

The derivative map of $\phi$ along the $M$ direction gives a cocycle

$$
(\omega, x, k) \rightarrow D_{x} \phi_{\omega}^{(k)}
$$

from $\Omega \times M \times \mathbb{N}$ to $G L(m, R)$ where $m=\operatorname{dim} M$.

Definition 1. For each $\omega \in \Omega, x \in M$ and $v \in T_{x} M$, we say that

$$
\lambda(\omega, x, v)=\lim _{k \rightarrow \infty} \frac{1}{k} \log \left(\left|D_{x} \phi_{\omega}^{(k)}(v)\right|\right),
$$

if the limit exists, is the fibrewise Lyapunov exponent associated to the point $(\omega, x)$ and the vector $v$.

By Oseledec's theorem (see [12, 13]) the limit exists for $\nu$-almost all $(\omega, x)$ for any $F$-invariant probability measure $\nu$ and therefore for a.e. $(\omega, x)$ there are real numbers $\lambda_{1}(\omega, x) \leq \lambda_{2}(\omega, x) \leq \cdots \leq \lambda_{m}(\omega, x)$ which are the fibrewise Lyapunov exponents corresponding to different directions in $T_{x} M$. If $\nu$ is ergodic, these 
numbers are constant $\nu$ almost everywhere and we denote them as $\lambda_{1}(\nu) \leq \cdots \leq$ $\lambda_{m}(\nu)$.

\subsection{Random expanding maps.}

1.2.1. Positive Lyapunov exponents. In this paper we shall be particularly interested in the case in which the fibrewise Lyapunov exponents are positive. Notice that in this case the definition implies that for all $\varepsilon>0$ sufficiently small, there exists a constant $C(\varepsilon, \omega, x)>0$ such that

$$
\left|D_{x} \phi_{\omega}^{(k)}(v)\right| \geq C(\varepsilon, \omega, x) e^{(\lambda(\omega, x, v)-\varepsilon) n}|v|
$$

for all $n \geq 1$. In particular, for an ergodic $F$-invariant measure $\nu$ with all fibrewise Lyapunov exponents positive, $\lambda_{m}(\nu) \geq \cdots \geq \lambda_{1}(\nu)=: \lambda(\nu)>0$. This implies that, for $\nu$ almost all $(\omega, x)$ and for all sufficiently small $\varepsilon>0$, there exists a constant $C(\varepsilon, \omega, x)>0$ such that

$$
\left|D_{x} \phi_{\omega}^{(k)}(v)\right| \geq C(\varepsilon, \omega, x) e^{(\lambda-\varepsilon) n}|v| .
$$

1.2.2. Random uniform expansion. In certain cases such an expansion estimate actually extends to all of $M$ with a constant $C$ independent of the point $x$.

Definition 2. A random map $F$ is called random uniformly expanding if there exists a constant $\lambda>0$ and a tempered random variable $C(\omega)>0$ such that for $\mathbb{P}$-almost all $\omega \in \Omega$ and every $x \in M$ we have

$$
\left\|D_{x} \phi_{\omega}^{n}(v)\right\| \geq C(\omega) e^{\lambda n}\|v\| .
$$

Notice that this extends the usual definition of a uniformly expanding map to the random setting by requiring the expansion rate $\lambda$ to be uniform in both $\omega$ and $x$ though still allowing the constant $C$ to depend (in a controlled way) on $\omega$. We recall that a random variable $g: \Omega \rightarrow \mathbb{R}^{+}$is tempered if

$$
\lim _{n \rightarrow \infty} \frac{1}{n} \log g\left(\theta^{n}(\omega)\right)=0, \mathbb{P}-\text { a.s. }
$$

Our first result says that such a uniform expansion property actually follows from an a priori weaker assumption.

Theorem 1. Let $F$ be a random map and suppose that all fibrewise Lyapunov exponents are positive for all measures $\nu \in \mathcal{E}_{\mathbb{P}}(F)$. Then $F$ is random uniformly expanding.

We emphasize that in our case the Lyapunov exponents are not assumed to be uniformly bounded away from 0 . Thus, a priori, we only have that for every $(\omega, x)$ in a subset of $\Omega \times M$ of full probability, i.e. in a set which has full measure for every invariant probability measure, there are constants $C(\omega, x)>0$ and $\lambda(\omega, x)>0$ such that $\left|D_{x} \phi_{\omega}^{(k)}(v)\right| \geq C(\omega, x) e^{\lambda(\omega, x) n}|v|$. Theorem 1 says that the expansion estimates actually hold for every $x$ and for constants $C, \lambda$ independent of $x$, and thus in particular that all fibrewise Lyapunov exponents are uniformly bounded away from 0 . 
1.2.3. Deterministic case. We remark that the results are nontrivial even in the special case in which the $\theta$-invariant measure $\mathbb{P}$ is a Dirac- $\delta$ measure supported on a single fixed point $\{p\}$. The setting stated above then reduces to the case in which $F: M \rightarrow M$ is a standard deterministic dynamical system, and an analogous result has been proved in [1, 4, 5]. The theorem we prove here represents a significant generalization of these results and is obtained by a different argument. The general question of the uniformity of in principle nonuniform functions has also been addressed in various contexts in other papers such as [15, 14].

1.2.4. Uniform bounds for expansion rates. As an immediate corollary of Theorem 1 we get the following statement.

Corollary 1. Let $F$ be a random map and suppose that there exist tempered random variables $C(\omega)>0$ and $\lambda(\omega)$ with $\int \log \lambda d \mathbb{P}>0$ such that for $\mathbb{P}$ almost all $\omega \in \Omega$ and every $x \in M$ we have

$$
\left\|D_{x} \phi_{\omega}^{n}(v)\right\| \geq C(\omega) \lambda^{(n)}(\omega)\|v\|
$$

where $\lambda^{(n)}(\omega)=\lambda(\omega) \cdots \lambda\left(\theta^{n-1} \omega\right)$. Then $F$ is random expanding. In particular $\lambda(\omega)>1$ can be chosen constant.

Proof. The assumption that $\int \log \lambda d \mathbb{P}>0$ then implies that for all measures $\nu \in$ $\mathcal{E}_{\mathbb{P}}(F)$ all fibrewise Lyapunov exponents are positive. Then Theorem 1 implies the result.

1.3. Random hyperbolic maps. We now state versions of these results for the cases in which $\theta$ is an invertible transformation and $\phi_{\omega}$ is a $C^{1}$ diffeomorphism for a.e. $\omega$.

\subsubsection{Random compact sets.}

Definition 3. $\Lambda=\{\Lambda(\omega): \omega \in \Omega\}$ is a random compact set if

(1) $\Lambda(\omega) \subset M$ is compact for a.e. $\omega$;

(2) $(x, \omega) \rightarrow d(x, \Lambda(\omega))$ is measurable.

Here $d$ is the Hausdorff distance on $M$. A random compact nonempty set $\Lambda=$ $\{\Lambda(\omega): \omega \in \Omega\}$ is invariant under $F$ if

$$
\phi_{\omega} \Lambda(\omega)=\Lambda(\theta \omega)
$$

for a.e. $\omega \in \Omega$.

\subsubsection{Random uniform hyperbolicity.}

Definition 4. A random, compact, $F$-invariant, nonempty set $\Lambda$ has a uniform tangent bundle splitting if there exist i) an open set $V$ with a compact closure $\bar{V}$, ii) a tempered random variable $\alpha>0$ with $\int \log \alpha d \mathbb{P}<\infty$, and iii) subbundles $\Gamma^{1}(\omega)$ and $\Gamma^{2}(\omega)$ of the tangent bundle $T \Lambda(\omega)$, depending measurably on $\omega$ and continuously on $x$, such that

(1) There exist a measurable family of open set $U(\omega)$ such that

(a) $\{x: d(x, \Lambda(\omega))<\alpha(\omega)\} \subset U(\omega) \subset V$;

(b) $\phi_{\omega} U(\omega) \subset V$;

(c) $\phi_{\omega}$ restricted to $U(\omega)$ is a diffeomorphism;

(d) $\int \log ^{+} \sup _{x \in U(\omega)}\left|D_{x} \phi_{\omega}\right| d \mathbb{P}<\infty$ and $\int \log ^{+} \sup _{x \in U(\omega)}\left|D_{x} \phi_{\omega}^{-1}\right| d \mathbb{P}<$ $\infty$. 
(2) (a) $T \Lambda(\omega)=\Gamma^{1}(\omega) \oplus \Gamma^{2}(\omega)$;

(b) $D \phi_{\omega} \Gamma^{1}(\omega)=\Gamma^{1}(\theta \omega)$ and $D \phi_{\omega} \Gamma^{2}(\omega)=\Gamma^{2}(\theta \omega)$;

(c) $\angle\left(\Gamma^{1}(\omega), \Gamma^{2}(\omega)\right) \geq \alpha(\omega)$, for a.e. $\omega$.

Definition 5 ([6]). A random, compact, $F$-invariant, nonempty set $\Lambda$ is a random uniformly hyperbolic set if it has a uniform tangent bundle splitting and there exists a constant $\lambda>0$ and a tempered random variable $C>0$ such that for a.e. $\omega$ and every $n \in \mathbb{N}$ we have

$$
\begin{aligned}
\left|D \phi_{\omega}^{(n)} \xi\right| \leq C(\omega) e^{\lambda n}|\xi| \quad \text { for } \quad \xi & \in \Gamma^{1}(\omega) \\
& \text { and }\left|D \phi_{\omega}^{(-n)} \eta\right| \leq C(\omega) e^{\lambda n}|\eta| \text { for } \eta \in \Gamma^{2}(\omega) .
\end{aligned}
$$

Theorem 2. Let $\Lambda$ be a random, compact, $F$-invariant, nonempty set with a uniform tangent bundle splitting, and suppose that for all measures $\nu \in \mathcal{E}_{\mathbb{P}}(F)$, all fibrewise Lyapunov exponents restricted to $\Gamma^{1}$ are negative and all fibrewise Lyapunov exponents restricted to $\Gamma^{2}$ are positive. Then $\Lambda$ is a random uniformly hyperbolic set for $F$.

Once again, we emphasize that this result is about showing that nonzero Lyapunov exponents on a full probability set actually imply uniform hyperbolicity and thus, in particular, that all Lyapunov exponents are actually uniformly bounded away from zero.

Theorem 2 follows immediately from Theorem 1 applied to each of the subbundles independently. In what follows we therefore assume the setup and assumptions of Theorem 1 .

\section{INVARIANT MEASURES ON THE UNIT TANGENT BUNDLE}

Let $S M=\{(x, v) \in T M:|v|=1\}$ denote the unit tangent bundle over $M$ and define the induced skew-product tangent map

$$
\widehat{T F}: \Omega \times S M \rightarrow \Omega \times S M
$$

by

$$
\widehat{T F}(\omega, x, v)=\left(\theta(\omega), \phi_{\omega}(x), \frac{D_{x} \phi_{\omega}(v)}{\left|D_{x} \phi_{\omega}(v)\right|}\right) .
$$

Since $\phi_{\omega}$ is a $C^{1}$ local diffeomorphism, the denominator in the definition above never vanishes and hence this map is well defined for all $(\omega, x, v) \in \Omega \times S M$. Extending the notation introduced above, we let $\operatorname{Pr}(S M)$ denote all probability measures supported on $S M$ and $\mathcal{M}_{\mathbb{P}}(\widehat{T F})$ denote all $\widehat{T F}$-invariant probability measures on $\Omega \times S M$ whose marginal on $\Omega$ coincide with $\mathbb{P}$ and let $\mathcal{E}_{\mathbb{P}}(\widehat{T F}) \subset \mathcal{M}_{\mathbb{P}}(\widehat{T F})$ denote the subsets of ergodic measures. Since $S M$ is compact, $\mathcal{M}_{\mathbb{P}}(\widehat{T F})$ is compact in the weak-star topology. Let

$$
\pi: \Omega \times S M \rightarrow \Omega \times M
$$

be the projection onto $\Omega \times M$. We have $\pi \circ \widehat{T F}=F \circ \pi$, and so if $m \in \mathcal{M}_{\mathbb{P}}(\widehat{T F})$, then $\pi^{*} m=m \circ \pi^{-1} \in \mathcal{M}_{\mathbb{P}}(F)$. Thus $\pi^{*}$ defines a map

$$
\pi^{*}: \mathcal{M}_{\mathbb{P}}(\widehat{T F}) \rightarrow \mathcal{M}_{\mathbb{P}}(F) .
$$

Lemma 1. $\pi^{*}\left(\mathcal{E}_{\mathbb{P}}(\widehat{T F})\right) \subset \mathcal{E}_{\mathbb{P}}(F)$. 
Proof. Let $A$ be a measurable set which is $F$ invariant. Then $\pi^{-1} A$ is a $\widehat{T F}$ invariant set. Since $m$ is ergodic, $m\left(\pi^{-1} A\right)=0$ or 1 . Thus $\nu(A)=\pi^{*} m(A)=m\left(\pi^{-1} A\right)=0$ or 1 . So $\nu$ is ergodic.

\section{UNIFORMLY POSITIVE LYAPUNOV EXPONENTS}

We define the random continuous function $\Phi: \Omega \times S M \rightarrow R$ by

$$
\Phi(\omega, x, v)=\log \left|D_{x} \phi_{\omega}(v)\right| .
$$

Lemma 2. There exists a measure $m^{*} \in \mathcal{M}_{\mathbb{P}}(\widehat{T F})$ such that

$$
\min _{m \in \mathcal{M}_{\mathbb{P}}(\widehat{T F})} \int_{\Omega \times S M} \Phi d m=\int_{\Omega \times S M} \Phi d m^{*}=: \Lambda>0 .
$$

In particular, all the fibrewise Lyapunov exponents of all invariant measures are uniformly bounded away from 0.

Proof. The existence of a minimizing measure $m^{*}$ follows immediately from the fact that $\mathcal{M}_{\mathbb{P}}(\widehat{T F})$ is compact and by noticing that $\Phi$ is a random continuous function on $\Omega \times S M$ and therefore $\int \Phi d m$ is continuous function on $\mathcal{M}_{\mathbb{P}}(\widehat{T F})$. Therefore it only remains to show that $\int \Phi d m^{*}>0$ or, equivalently, that $\int \Phi d m>0$ for any $m \in \mathcal{M}_{\mathbb{P}}(\widehat{T F})$. Moreover, by the Ergodic Decomposition Theorem we can assume without loss of generality that $m$ is ergodic.

Thus let $m \in \mathcal{E}_{\mathbb{P}}(\widehat{T F})$ and $\nu=\pi^{*} m \in \mathcal{E}_{\mathbb{P}}(F)$. Notice that $\pi$ maps full measure sets for $m$ to full measure sets for $\nu$. By Birkhoff's Ergodic Theorem we have, for $m$ almost every $(\omega, x, v)$,

$$
\int_{\Omega \times S M} \Phi(\omega, v) d m=\lim _{n \rightarrow \infty} \frac{1}{n} \sum_{i=0}^{n-1} \Phi\left((\widehat{T F})^{i}(\omega, x, v)\right) .
$$

By the definition of $\Phi$ we have

$$
\sum_{i=0}^{n-1} \Phi\left((\widehat{T F})^{i}(\omega, x, v)\right)=\log \left|D_{x} \phi_{\omega}^{(n)}(v)\right|,
$$

and therefore

$$
\lim _{n \rightarrow \infty} \frac{1}{n} \sum_{i=0}^{n-1} \Phi\left((\widehat{T F})^{i}(\omega, x, v)\right)=\lim _{n \rightarrow \infty} \frac{1}{n} \log \left|D_{x} \phi_{\omega}^{(n)} v\right| .
$$

Applying Birkhoff's Theorem again, the limit on the right converges to $\lambda(\omega, x, v)$, which is $>0$ by our assumptions that all fibrewise Lyapunov exponents are positive.

\section{UNIFORM HYPERBOLICITY}

In the previous section we showed that all Lyapunov exponents are uniformly bounded away from zero. We now need to extend the corresponding expansion estimates to every point $x \in M$.

Lemma 3. For any $\Lambda>\lambda>0$ we have that for a.e. $\omega$ there exists a constant $C(\omega)>0$ such that for all $x \in M, v \in T_{x} M$, and $n \geq 1$

$$
\left|D_{x} \Phi_{\omega}^{(n)} v\right| \geq C(\omega) e^{\lambda n}|v|
$$


Notice that this is not quite the end result, since we still need to prove that $C(\omega)$ is tempered. We shall do this in the next section.

Proof. The statement follows immediately from the fact that for a.e. $\omega$ we have

$$
\lim _{n \rightarrow \infty} \frac{1}{n} \min _{(x, v) \in S M}\left\{\log \left|D_{x} \phi_{\omega}^{(n)} v\right|\right\}=\Lambda .
$$

To prove this, we show first of all that the limit exists and is independent of $\omega$; then we show that it is equal to $\Lambda$.

Existence of the limit. To get the existence of the limit, let

$$
A_{n}(\omega)=\min _{(x, v) \in S M} \log \left|D_{x} \phi_{\omega}^{(n)} v\right| .
$$

Then $A_{n+m}(\omega) \geq A_{n}(\omega)+A_{m}\left(\theta^{n} \omega\right)$. Therefore the sequence $\left\{A_{n}\right\}$ is supadditive, the sequence $\left\{-A_{n}\right\}$ is subadditive and, from the subadditive ergodic theorem [9] and the ergodicity of $\mathbb{P}$ there exists a constant $A$ such that

$$
\lim _{n \rightarrow \infty} \frac{1}{n} \min _{(x, v) \in S M}\left\{\log \left|D_{x} \phi_{\omega}^{(n)} v\right|\right\}=A
$$

for a.e. $\omega$.

Upper bound. From the previous section we know that $n^{-1} \log \left|D_{x} \phi_{\omega}^{(n)} v\right|$ converges to $\Lambda$ for some points (indeed, a set of points of full measure for the minimizing measure $m^{*}$ ), and therefore we must have $A \leq \Lambda$.

Lower bound. It therefore only remains to prove $A \geq \Lambda$. Suppose by contradiction that $A<\Lambda$. We will show that this implies that there is a measure $\mu$ for which

$$
\int \Phi d \mu<\Lambda
$$

which gives a contradiction.

Construction of the measure $\mu$. Notice first of all that, since $n^{-1} A_{n} \rightarrow A$ for a.e. $\omega$, we can choose a set $U$ of arbitrarily large measure on which this convergence is uniform. From this and the definition of $A_{n}$, for every $\epsilon>0$ there exists $N>0$ such that for all $n \geq N$ there exists a measurable function $\omega \mapsto\left(x_{n}(\omega), v_{n}(\omega)\right) \in S M$ defined in $U$ such that

$$
\frac{1}{n} \log \left|D_{x_{n}(\omega)} \phi_{\omega}^{(n)} v_{n}(\omega)\right|=\frac{1}{n} A_{n}(\omega)<A+\epsilon .
$$

To see this, just consider the weakly measurable and closed valued set function

$$
w \mapsto\left\{(x, v) \in S M: \log \left|D_{x} \phi_{w}^{(n)} v\right| \text { is minimal }\right\},
$$

defined in $U$, and choose any measurable selection $\left(x_{n}(\omega), v_{n}(\omega)\right)$ (for the existence of such a selection see, for instance, Theorem 4.1 in [16]). Then, for each $\omega \in U$ and each $n \geq 1$ we define a probability measure $\sigma_{n}(\omega)=\delta_{x_{n}(\omega), v_{n}(\omega)}$, where $\delta_{x, v}$ denotes the Dirac-delta measure at the point $(x, v) \in S M$. We also let $G=\bigcup_{i=-\infty}^{\infty} \theta^{i}(U)$ (notice that ergodicity implies that $\mathbb{P}(G)=1$ ), and for $\omega \in G \backslash U$ define $\sigma_{n}(\omega) \equiv \delta_{x, v}$ for some arbitrary point $(x, v) \in S M$ which can be chosen independently of $\omega$ or $n$. We can now define probability measures

$$
\mu_{n}(\omega)=\frac{1}{n} \sum_{i=0}^{n-1}\left((\widehat{T F})^{i} \sigma_{n}\right)(\omega) .
$$


It is easy to prove that the marginal of $\mu_{n}$ on $\Omega$ coincides with $\mathbb{P}$, and it is well known that $\left\{\mu_{n}\right\}$ has a subsequence converging to an invariant measure $\mu \in \mathcal{M}_{\mathbb{P}}(\widehat{T F})$ (see Arnold [3]). Without loss of generality, we suppose $\lim _{n \rightarrow \infty} \mu_{n}=\mu$.

Contradiction. It remains to prove (2), i.e. $\int_{\Omega \times S M} \Phi d \mu<\Lambda$, to get the desired contradiction. By the continuity of $\Phi$ we have

$$
\int_{\Omega \times S M} \Phi d \mu=\lim _{n \rightarrow \infty} \int_{\Omega \times S M} \Phi d \mu_{n}
$$

By the definition of $\mu_{n}$ we have

$$
\int_{\Omega \times S M} \Phi d \mu_{n}=\frac{1}{n} \sum_{i=0}^{n-1} \int_{\Omega \times S M} \Phi d\left((\widehat{T F})^{i} \sigma_{n}\right)(\omega) d \mathbb{P},
$$

and by the definition of $\sigma_{n}$ and the fact that $\mathbb{P}(G)=1$, the right hand side above is equal to

$$
\frac{1}{n} \int_{U} \sum_{i=0}^{n-1} \Phi\left((\widehat{T F})^{i}\left(\omega, x_{n}(\omega), v_{n}(\omega)\right)\right) d \mathbb{P}+\frac{1}{n} \int_{G \backslash U} \sum_{i=0}^{n-1} \Phi\left((\widehat{T F})^{i}(\omega, x, v)\right) d \mathbb{P} .
$$

It is therefore sufficient to consider the limits of these two integrals and show that their sum is strictly less than $\Lambda$. To bound the first part notice that

$$
\sum_{i=0}^{n-1} \Phi\left((\widehat{T F})^{i}\left(\omega, x_{n}(\omega), v_{n}(\omega)\right)\right)=\log \left|D_{x_{n}(\omega)} \phi_{\omega}^{(n)} v_{n}(\omega)\right|,
$$

and therefore (3) gives an upper bound of $A+\varepsilon$. For the second we have

$$
\begin{aligned}
\int_{G \backslash U} \sum_{i=0}^{n-1} \Phi\left((\widehat{T F})^{i}(\omega, x, v)\right) d \mathbb{P} & \leq \sum_{i=0}^{n-1} \int_{G \backslash U}\left|D \phi_{\theta^{i}(\omega)}\right| d \mathbb{P} \\
& =\sum_{i=0}^{n-1} \int_{\theta^{-i}(G \backslash U)}\left|D \phi_{\omega}\right| d \mathbb{P} .
\end{aligned}
$$

Recall that $\left|D \phi_{\omega}\right|=\max _{(x, v)}\left|D_{x} \phi_{\omega}(v)\right|$. Since $\theta: \Omega \rightarrow \Omega$ is an invertible transformation preserving the ergodic measure $\mathbb{P}$, we have $\mathbb{P}\left(\theta^{-i}(G \backslash U)\right)=\mathbb{P}(G \backslash U)<\delta$. Thus, by the integrability condition on $\left|D \phi_{\omega}\right|$ we can choose $U$ of sufficiently large measure so that

$$
\int_{\theta^{-i}(G \backslash U)}\left|D \phi_{\omega}\right| d \mathbb{P}<\epsilon .
$$

Since $\epsilon$ is arbitrary we get the desired contradiction.

\section{TEMPERED RANDOM VARIABLES}

Finally, it only remains to show that that "constant" $C(\omega)$ is a tempered random variable. To see this, notice first of all that we can choose

$$
C(\omega)=\inf _{n \geq 1}\left\{e^{-\lambda n} \min _{(x, v) \in S M}\left|D_{x} \phi_{\omega}^{(n)}(v)\right|\right\} .
$$

Then we have

Lemma 4. $C(\omega)$ is a tempered random variable. 
Proof. We want to compare $C(\theta \omega)$ to $C(\omega)$. Let $D_{n}(\omega):=\min _{(x, v) \in S M}\left|D_{x} \phi_{\omega}^{(n)}(v)\right|$. Then

$$
\frac{C(\theta \omega)}{C(\omega)}=\frac{\inf _{n \geq 1}\left\{e^{-\lambda n} D_{n}(\theta \omega)\right\}}{\inf _{n \geq 1}\left\{e^{-\lambda n} D_{n}(\omega)\right\}}=\frac{\min \left\{e^{-\lambda} D_{1}(\theta \omega), \inf _{n \geq 2}\left\{e^{-\lambda n} D_{n}(\theta \omega)\right\}\right\}}{\min \left\{e^{-\lambda} D_{1}(\omega), \inf _{n \geq 2}\left\{e^{-\lambda n} D_{n}(\omega)\right\}\right\}} .
$$

We consider two cases. Suppose first that $C(\omega)=e^{-\lambda} D_{1}(\omega) \leq \inf _{n \geq 2}\left\{e^{-\lambda n} D_{n}(\omega)\right\}$. Then

$$
\frac{C(\theta \omega)}{C(\omega)} \leq \frac{D_{1}(\theta \omega)}{D_{1}(\omega)}
$$

On the other hand, suppose that $C(\omega)=\inf _{n \geq 2}\left\{e^{-\lambda n} D_{n}(\omega)\right\} \leq e^{-\lambda} D_{1}(\omega)$. Then, keeping in mind that $D_{n}(\omega)=D_{n-1}(\theta \omega) D_{1}(\omega)$, we have, for any $n \geq 2$,

$$
e^{-\lambda n} D_{n}(\omega) \geq e^{-\lambda(n-1)} D_{n-1}(\theta \omega) e^{-\lambda} D_{1}(\omega) \geq C(\theta \omega) e^{-\lambda} D_{1}(\omega) .
$$

Hence $C(\omega) \geq C(\theta \omega) e^{-\lambda} D_{1}(\omega)$ and so, combining the estimates in the two cases, we have

$$
\frac{C(\theta \omega)}{C(\omega)} \leq \max \left\{\frac{D_{1}(\theta \omega)}{D_{1}(\omega)}, \frac{e^{\lambda}}{D_{1}(\omega)}\right\} \leq \frac{\max \left\{\left|D \phi_{\theta \omega}\right|, e^{\lambda}\right\}}{D_{1}(\omega)} .
$$

Since $1 / D_{1}(\omega) \leq\left|D \phi_{\omega}^{-1}\right|$ the integrability assumptions (11) imply that

$$
\log ^{+} \frac{C(\theta \omega)}{C(\omega)} \in L^{1}(\Omega, \mathcal{F}, \mathbb{P})
$$

where $\log ^{+} a=\max \{\log a, 0\}$. The statement in the lemma then follows by a standard general result that any positive finite measurable function $g$ such that $\log ^{+} \frac{g(\theta(\omega))}{g(\omega)} \in L^{1}(\Omega, \mathcal{F}, \mathbb{P})$ is tempered. For completeness we give a proof here. By the subadditive ergodic theorem the following limit exists for a.e. $\omega$ :

$$
\lim _{n \rightarrow \infty} \frac{1}{n} \sum_{k=0}^{n-1} \log \frac{g \circ \theta^{k+1}}{g \circ \theta^{k}}=\lim _{n \rightarrow \infty} \frac{1}{n} \log \frac{g \circ \theta^{n}}{g}=\lim _{n \rightarrow \infty} \frac{1}{n} \log \left(g \circ \theta^{n}\right)=h .
$$

The last equality follows from the fact that $\lim _{n \rightarrow \infty} n^{-1} \log g=0$. By the definition of a tempered random variable, it is therefore sufficient to show that $h=0$ for a.e. $\omega$. For each fixed $\delta>0$ and using the invariance of the measure $\mathbb{P}$ for $\theta$, we have

$$
\begin{aligned}
\lim _{n \rightarrow \infty} \mathbb{P}\left(\left\{\omega: \frac{1}{n} \log \left|g \circ \theta^{n}(\omega)\right| \geq \delta\right\}\right) & =\lim _{n \rightarrow \infty} \mathbb{P}\left(\theta^{-n} g^{-1}\left(-e^{n \delta}, e^{n \delta}\right)^{c}\right) \\
& =\lim _{n \rightarrow \infty} \mathbb{P}\left(g^{-1}\left(-e^{n \delta}, e^{n \delta}\right)^{c}\right)=0 .
\end{aligned}
$$

This means that the sequence of functions $\frac{1}{n} \log \left(g \circ \theta^{n}\right)$ converges to 0 in measure and therefore some subsequence converges to 0 a.e. Since we know from the above that the sequence actually converges a.e., this yields the result.

\section{REFERENCES}

[1] Alves, J. F., Uniform hyperbolicity of some nonuniformly hyperbolic systems, Proc. Amer. Math. Soc., 131(4) (2003) 1303-1309. MR1948124 (2003k:37046)

[2] Arbieto, A.; Matheus, C.; Oliveira, K., Equilibrium states for random non-uniformly expanding maps, Nonlinearity, 17 (2004) 581-593. MR2039060 (2005c:37010)

[3] Arnold, L., Random dynamical systems (1998) Springer-Verlag. MR.1723992 (2000m:37087)

[4] Cao, Y., Nonzero Lyapunov exponents and uniform hyperbolicity, Nonlinearity, 16 (2003) 1473-1479. MR1986306 (2005g:37061)

[5] Cao, Y.; Rios, I.; Luzzatto, S., A minimum principle for Lyapunov exponents and a higherdimensional version of a theorem of Mané, Qualitative Theory of Dynamical Systems, 5 (2004) 261-273. MR2275440(2007k:37031) 
[6] Gundlach,V. M.; Kifer,Y., Random hyperbolic systems, Stochastic dynamics (Bremen, 1997), 17-145, Springer, New York, 1999. MR1678467 (2000b:37053)

[7] Khanin, K.; Kifer, Y., Thermodynamic formalism for random transformations and statistical mechanics, Sinai's Moscow Seminar on Dynamical Systems, Amer. Math. Soc. Transl. Ser. 2, 171, 107-140, Amer. Math. Soc., Providence, RI, 1996. MR1359097 (96j:58136)

[8] Kifer, Y., Random dynamics and its applications, Proceedings of the International Congress of Mathematicians, Vol. II (Berlin, 1998), Doc. Math., 1998, Extra Vol. II, 809-818 (electronic). MR:1648128 (99k:58113)

[9] Kingman, J., Subadditive ergodic theory, Annals of Probability, 1 (1973) 883-904. MR 0356192 (50:8663)

[10] Liu, P. D., Dynamics of random transformations: smooth ergodic theory, Ergodic Theory Dynam. Systems, 21 (2001) 1279-1319. MR1855833 (2002g:37024)

[11] Liu, P. D.; Zhao, Y., Large deviations in random perturbations of Axiom A basic sets, J. London Math. Soc, 68 (2003) 148-164. MR1980249 (2006j:37059)

[12] Oseledec, V. I., A multiplicative ergodic theorem. Characteristic Ljapunov, exponents of dynamical systems, Transactions of the Moscow Mathematical Society, 19, Amer. Math. Soc., Providence, RI, 1968. MR0240280 (39:1629)

[13] Ruelle, D., Characteristic exponents and invariant manifolds in Hilbert space, Ann. of Math. (2), 115 (1982) 243-290. MR647807 (83j:58097)

[14] Slomczyński, W., Subadditive ergodic theorems in $C(X)$, Ital. J. Pure Appl. Math. (1997) 17-28. MR1666986 (99m:47008)

[15] Sturman, R.; Stark, J., Semi-uniform ergodic theorems and applications to forced systems, Nonlinearity, 13 (2000) 113-143. MR1734626 (2000m:37041)

[16] Wagner, D., Survey of measurable selection theorems, SIAM J. Control and Optimization, 15 (1977) 859-903. MR0486391(58:6137)

Department of Mathematics, Suzhou University, Suzhou 215006, Jiangsu, People's Republic of China

E-mail address: ylcao@suda.edu.cn

Department of Mathematics, Imperial College, 180 Queen's Gate, London SW7 2AZ, UNITED KingDOM

E-mail address: Stefano.Luzzatto@imperial.ac.uk

Department of Mathematics, Universidade Federal Fluminense, Niteroi, Rio de JANEIRO, BRAZIL

E-mail address: rios@mat.uff.br 